УДК 697.331

\title{
М.О. Прусенков
}

Одеська державна академія будівництва і архітектури, вул. Дідріхсона, 4, м. Одеса, 65029

\section{СХЕМА ВИЗНАЧЕННЯ СКЛАДОВИХ ВТРАТ ТЕПЛА У РУХОМОМУ ШАРІ БАГАТО- ШАРОВИХ ОГОРОДЖУЮЧИХ КОНСТРУКЦІЙ}

Зміни ставлення нормативної бази до рухомих шарів конструкиії огорожі, вимагає перегляду розрахункової схеми визначення втрат потоком, який перетинає ї̈ поперек. Наведена схема сприяє розробиі способу уникнення втрат, які виникають через різницю температур на поверхнях дотичних шарів, щуо автоматично виключалося 6 огорожах, які складалися лише з замкнутих шарів. Вона ілюструє вирівнювання температурна межах шарів надходженнями тепла у складовій, що рухається у рухомому шарі. Слід додати до опису способу оцінку енергообміну у рухомому шарі, але ие виходить за рамки мети публікаиії.

Ключові слова: рухливий та замкнутий шари - замкнена складова потоку - теплообмін - втрати енергії.

\section{Н.А. Прусенков}

Одесская государственная академия строительства и архитектуры, ул. Дидрихсона, 4, г. Одесса, 65029

\section{СХЕМА ОПРЕДЕЛЕНИЯ СОСТАВЛЯЮЩИХ ПОТЕРЬ ТЕПЛА В ПОДВИЖНОМ СЛОЕ МНОГОСЛОЙНЫХ ОГРАЖДАЮЩИХ КОНСТРУКЦИЙ}

\begin{abstract}
Изменение отношения нормативной базы к подвижности слоев ограждающих конструкиий требует пересмотра расчетной схемь для определения потерь потоком, переходящим ее. Представленная схема благоприятствует разработке способа избежания потер, возникаюших из-за разности температур на поверхностях соприкасающихся слоев, что автоматически исключалось в ограждениях, состоящих только из замкнутых слоев. Она иллюстрирует выравнивание температур на границах слоев поступлениями тепла в подвижной составляющей подвижного потока. Следует дополнить описание способа оценкой энергообмена в пределах подвижного слоя, что выходит за рамки ичели публикациии.

Ключевые слова: подвижный и замкнутый слои - составляющая потока - теплообмен- потери энергии.
\end{abstract}

This work is licensed under the Creative Commons Attribution International License (CC BY). http://creativecommons.org/licenses/by/4.0/

\section{I. ВВЕДЕНИЕ}

Действующие нормы теплоизоляции зданий ДБН В.2.6-31:2006, с изменениями [1], регламентирующие требования к многослойным ограждающим конструкциям (МОК), не предусматривают возникновения потерь энергии на теплообмен изза разности температур на поверхностях соприкасающихся слоев. Для МОК, состоящих исключительно из замкнутых слоев, такая проблема не возникает. Перспективы совершенствования конструкций ограждений прогнозируют создание МОК, в которых использованы источники и устройства, изменяющие температуру отдельных слоев и их поверхностей. Возникшие при этом перепады температур на поверхностях слоев могут стать причиной возникновения потоков между ними, стимулирующих появление потерь энергии на осуществление теплообмена между ними. Сле- дует предусмотреть способ регулирования потоков энергии в МОК, при поступлениях в отдельные слои. Цель публикации - создание расчетной схемы МОК, учитывающей изменение температур на соприкасающихся поверхностях слоев и предусматривающей возможность исключения потерь на перетоки энергии.

\section{ІІ. ПРЕДПОСЫЛКИ УСТРОЙСТВА ПО- ДВИЖНОГО СЛОЯ В МОК}

Для определения затрат энергии системами отопления и вентиляции на поддержание заданного перепада температур в помещении учитывают теплопередачу вентиляционных потоков ограждаемых помещений в период эксплуатации. При этом, включения расчетов, соответствующих потерь энергии в число затрат теплоизоляционной оболочки сооружения $\left(Q_{k}\right.$ и $q_{\text {зд }}$ см. Прил. Н к ДБН 
[1] ф.Н 1-6), допустимы для определения потерь энергии тепловым потоком через МОК с существенными оговорками и уточнениями. Важнейшим достижением указанного приложения следует считать «скрытую» попытку узаконить учет потерь энергии подвижными потоками, переходящими через МОК в период эксплуатации. Наличие этого приложения к действующей ДБН [1] стимулирует поиск путей и способов снижения потерь энергии потоками, пересекающими МОК в период эксплуатации, что является целью данной публикации. Все вышеизложенное приведено специально для того, чтобы предупредить и предотвратить возможную путаницу между характеристиками, необходимыми для расчета и проектирования МОК и данными, используемыми действующей ДБН для определения удельных теплозатрат на отопление всего здания.

С другой стороны, декларируя принцип постоянства удельных термических сопротивлений слоев MOK $(R x=\mathrm{const})$, закрепляемый при проектировании неизменностью конструктивов:

- $\delta_{\mathrm{x}}=$ const - толщина слоя ограждения;

$-\lambda_{\mathrm{x}}=$ const - теплопроводность слоя, вт $/\left(\mathrm{M}^{2} \times{ }^{\circ} \mathrm{C}\right)$, действующая норма оговаривает и утверждает существование в МОК только замкнутых слоев (см. прил. Б к ДБН [1]), неизменных в период эксплуатации. Альтернативой выявленному противоречию является наличие в предшествующей норме СНиП 11-3-79** «Строительная техника» и действующей ДБН [1] одинаковых значений удельных термических сопротивлений для замкнутых воздушных потоков и их составляющих из подвижных сред. При этом, характеристики неподвижных (замкнутых) составляющих успешно используются для определения значений удельных термических сопротивлений воздушных (подвижных) потоков в вентилируемых фасадах [2]. Это прогнозирует перспективность использования существующих предпосылок учета свойств подвижных потоков и их составляющих для совершенствования теоретической базы расчета и регулирования теплопотерь энергии через МОК. Любой поток, переходящий через заданный слой МОК может быть разделен (дистрибутирован) на подвижную и замкнутую составляющие. Суммарный эффект (результат) проявления одновременности их действия в пределах слоев МОК существующие методики и нормативы не предусматривают, вынуждая обеспечить обнуление значений характеристик подвижной составляющей, обеспечивая выполнение постулата постоянства удельного термического сопротивления МОК и ее слоев на этапе эксплуатации [1].

Ссылаясь на общеизвестные примеры процессов пневмо- и гидротранспортирования справедливо выделить существование большого числа способов, использующих перемещение подвижных сред в каналах, которые допустимо представить слоями МОК. При этом подвижные потоки сред в них или их составляющие проявляют свойства, которые не предусмотрены и не используют- ся действующим нормативом [1] для регулирования потерь в потоках МОК - ввиду априорного исключения их из расчетов на этапе эксплуатации [4], как следствия декларированного ДБН постоянства удельного термического сопротивления. Теоретически, существование примеров использования свойств подвижных потоков в период эксплуатации опровергает объективность предпосылок исключения их из расчетов [5]. Не нарушая требований действующих норм [1] и учебнотеоретической литературы [2, 3] целесообразно предусмотреть возможность объяснения взаимодействия тепловых потоков, пересекающихся в общем (подвижном) слое МОК. Для удобства анализа и описания процессов, происходящих в подвижном слое МОК, поток, преодолевающий его, дистрибутирован на замкнутую и подвижную составляющие. На начальном этапе модернизации методики регулирования потерь энергии в МОК взаимодействием составляющих процесса перехода тепла достаточно ограничиться определением результирующих значений характеристик составляющих потоков на поверхностях слоев, воспользовавшись способом суперпозиционирования [5]. Эффект, проявляемый каждым из свойств взаимодействующих составляющих на границах слоев должен быть адекватен сумме их проявлений в общем для всех составляющих слое.

Один из простейших вариантов уменьшения потерь энергии потоком, пересекающим МОК это компенсация затрат замкнутой составляющей на переход потока через слои поступлением дополнительной энергии от внешних источников на этапе эксплуатации в его подвижной составляющей. Для его реализации необходимо предусмотреть возможность взаимодействия (теплообмена) поступлений в подвижной составляющей с затратами в замкнутой составляющей, пересекающимися в единой среде подвижного слоя. Создание такого слоя на этапе осуществления капитальных затрат, обеспечивающего пересечение в его среде подвижной и замкнутой составляющих, на которые он суперпозиционируется - необходимое условие возможности реализации предлагаемого способа регулирования потерь потоком, пересекающим МОК.

Подвижный слой - это канал в теле МОК (отдельный слой), окруженный твердыми поверхностями соседних замкнутых слоев, в котором может перемещаться энергонесущая среда, обеспечивающая взаимодействие его подвижной, переносящий энергию в своей среде, и замкнутой (согласно действующим нормам) составляющих в нем, на которые он суперпозиционирован.

Bсе перечисленные ранее в публикации нормативные источники [1-3] изобилуют рекомендациями, рекламирующими перспективность использования подвижных слоев и потоков в ограждениях, но исключающими учет изменения свойств на этапе эксплуатации. Возможность создания таких МОК подтверждается наличием большого числа примеров пневмотранспортных, 
отопительных и вентиляционных установок. Почти все задачи создания многослойных ограждающих конструкций следует считать давно известными. Они решены для достижения целей отопления, вентиляции и пневмотранспорта. Но, кроме того, теория определения характеристик взаимодействующих составляющих, использованная для достижения указанных целей может быть существенно дополнена учетом свойств подвижных составляющих, проявляемых при эксплуатации, для регулирования потерь энергии тепловым потоком, переходящим через ограждение, что исключено действующей нормой.

\section{III. ВКЛЮЧЕНИЕ ПОДВИЖНОГО СЛОЯ В СОСТАВ МОК}

Включение дополнительного подвижного слоя (пс) в состав конструкции МОК (в число слоев) требует выделение его из общего числа $-x$, как имеющего особые свойства, что обеспечивается разделением общего числа слоев, индексируемых в формулах - $(x)$ дополнением индексами (п - подвижный поток или 3 - замкнутый слой). На данном этапе обоснования необходимости включения в нормы методики расчета МОК с подвижным слоем рассмотрена ограждающая конструкция, состоящая из минимально возможного числа слоев, один из которых подвижный, с подвижной средой - всего три слоя:

- слой наружного ограждения - (индексируется - но), имеющий наружную $\left(t_{\text {нон }}\right)$ и внутреннюю $\left(t_{\text {нов }}\right)$ поверхности с соответствующими температурами. Его внутренняя поверхность соприкасается с наружной поверхностью подвижного слоя $\left(t_{\text {псн }}\right)$, а наружная - с окружающим пространством снаружи ограждения, температура которого $-t_{\mathrm{H},}$ с учетом потери теплоперехода $\left(\Delta t_{\mathrm{H}}\right)$ на наружной поверхности МОК;

- слой внутреннего материала стены - (индексируется - мс), имеющий наружную $\left(t_{\mathrm{McH}}\right)$ и внутреннюю $\left(t_{\mathrm{mcB}}\right)$ поверхности с соответствующими температурами. Его наружная поверхность соприкасается с внутренней поверхностью подвижного слоя $\left(t_{\text {псВ }}\right)$, а внутренняя - с окружающим пространством с внутренней стороны ограждения, температура которого $-\mathrm{t}_{\mathrm{B}}$, с учетом потери теплоперехода $\left(\Delta t_{\mathrm{B}}\right)$ на внутренней поверхности МОК;

- подвижный слой - (индексируется - пс), имеющий наружную $\left(t_{\text {псH }}\right)$ и внутреннюю $\left(\mathrm{t}_{\text {псв }}\right)$ поверхности, ограниченные поверхностями соприкасающихся замкнутых слоев. Температуры на поверхностях подвижного слоя предложено регулировать за счет поступлений от внешних источников в подвижной среде в период эксплуатации МОК. Это позволяет добиться снижения потерь энергии на переход тепла между соприкасающимися слоями МОК изменением параметров поступлений в подвижный слой. Его наружная поверхность соприкасается с внутренней поверхностью слоя наружного ограждения $\left(t_{\text {нов }}\right)$, а внутрен- няя поверхность соприкасается с наружной поверхностью материала стены $\left(t_{\text {мсH }}\right)$. Регулированием теплотехнических характеристик составляющих подвижного потока при эксплуатации достигают равенства температур поверхностей подвижного слоя, температурам соприкасающихся с соответствующими поверхностями замкнутых слоев, исключающего возникновение дополнительных потоков тепла между ними, за счет поступлений энергии от внешних источников в среде подвижной составляющей этого слоя.

\section{CХЕМА ИЗМЕНЕНИЯ ТЕМПЕРАТУРЫ В МОК С ПОДВИЖНЫМ СЛОЕМ}

Для обеспечения удобства разработки и пояснения способа регулирования потерь энергии в потоке, переходящем МОК с подвижным слоем приведена схема изменения температуры при переходе потоком тепла через многослойное ограждение с подвижным слоем (рисунок 1):

- на фоне поперечного сечения трехслойной ограждающей конструкции (сплошная линия 1,5 пт), для которого выносными линиями (0,3пт) указаны толщины слоев и всей МОК полностью:

- толщина наружного слоя (но) ограждения (замкнутого слоя) $-\delta_{\text {но }}$, м;

- толщина подвижного слоя (пс), среда которого движется (подвижного) $-\delta_{\text {пс }}$, м;

- толщина внутреннего слоя (мс) материала стены (замкнутого) $-\delta_{\text {мс }}$, м;

- общая толщина МОК - $\delta_{\text {огр }}$, м;

- оси прямоугольных координат (линия 0,5пт) $t\left({ }^{\circ} \mathrm{C}\right), x(\mathrm{M}), \mathrm{c}$ соответствующими масштабами и расстояниями между сравниваемыми значениями;

- ломаная кривая, составленная из отрезков с разными углами наклона (линия 3,0пт), являющихся геометрическими местами точек температур в слоях, которые являются функциями расстояний от соответствующих поверхностей МОК $t_{\mathrm{x}}=f\left(\delta_{\mathrm{x}}\right)$, классифицированных по характеристикам для каждого слоя;

- наклонный отрезок сплошной линии (1,0пт), соединяющий значения температур на внутренней и наружной границах подвижного слоя $\left(t_{\text {псв }}\right.$ и $\left.t_{\text {псн }}\right)$ и пересекающий этот слой. Именно достижение на концах его равенств температур, температурам на соответствующих наружной и внутренней поверхностях слоев, соприкасающихся с подвижным слоем $\left(t_{\mathrm{mcH}}=t_{\text {псВ }}\right.$ и $\left.t_{\text {псН }}=t_{\text {нов }}\right)$, обеспечивает исключение потерь применением способа регулирование потерь на остывание за счет поступлений тепла от внешних источников в подвижном слое $\left(\Delta t_{\text {пс }}=\Delta t_{\text {псВ }}-\Delta t_{\text {псН }}\right)$ и иллюстрирует идею использования свойств подвижных слоев;

- сплошная ломаная кривая в подвижном слое (линия 3,0пт), иллюстрирует существование подвижной и замкнутой составляющих, на которые позиционируют поток подвижного слоя, исключая 
дополнительные потери тепла на его поверхностях $\left(\Delta t_{\text {пс }}=\Delta t_{\text {пс } 3}+\Delta t_{\text {псП }}\right)$ :

- в замкнутой составляющей подвижного слоя согласно ДБН $-\Delta t_{\text {псз }}$ (сплошная наклонная линия); - в подвижной составляющей $-\Delta t_{\text {псп }}$ (сплошная вертикальная линия).

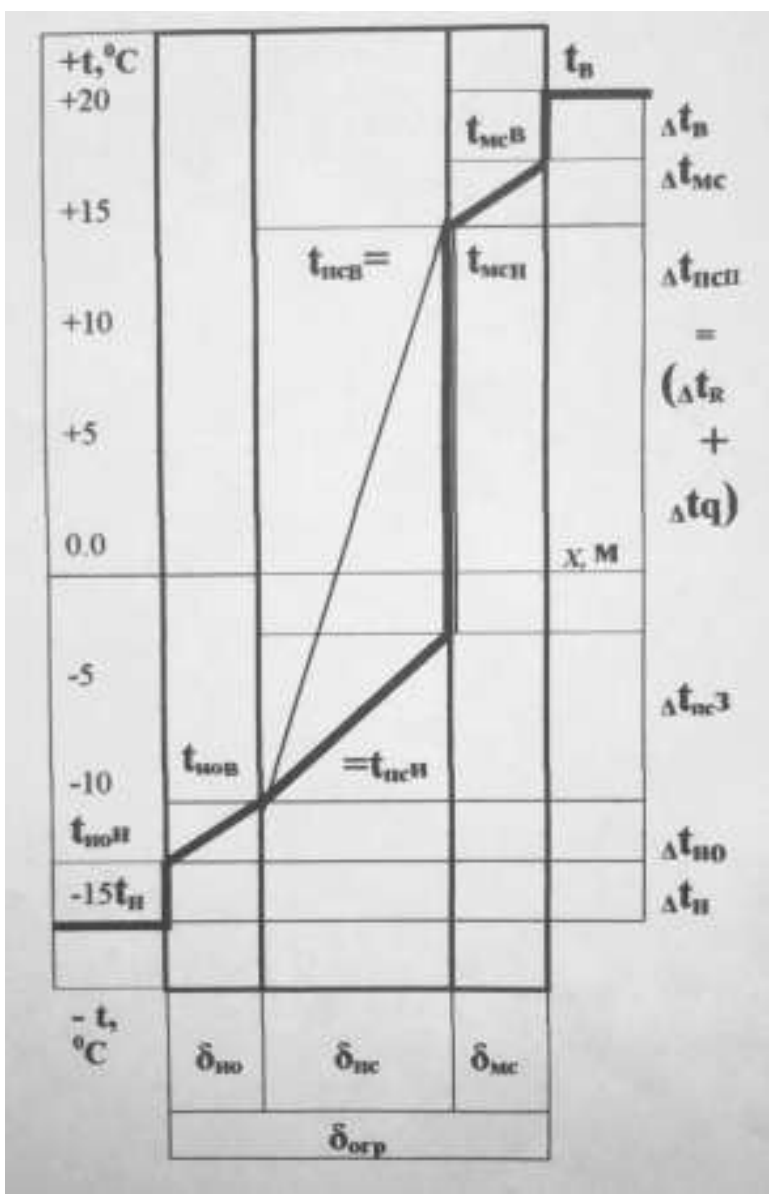

Рисунок 1 - Схема изменения температуры при переходе потоком тепла через многослойное ограждение с подвижным слоем

\section{Примечания к рисунку 1:}

1. Изменения перепадов температур на границах подвижного слоя $\left(\delta_{\text {пс }}\right)$ рассчитываются по формуле:

$$
\Delta t_{\text {пс }}=\left(\Delta t_{R}+\Delta t_{\mathrm{q}}\right)+\Delta t_{\text {пс } 3}=t_{\text {псВ }}-t_{\text {псН }}
$$

2. На схеме буквами обозначены поверхности, слои и составляющие затрат:

- В - внутренняя поверхность ограждения;

- Н - наружная поверхность ограждения

- мс-слой материала стены здания (замкнутый слой);

- пс - слой с подвижной средой (подвижный слой);

- но - слой наружного ограждения (замкнутый слой);
- $\Delta t_{R}$ - потери температуры замкнутых составляющих из-за малости $R,{ }^{\circ} \mathrm{C}$;

- $\Delta t_{\mathrm{q}}$ - затраты на уравнивание температур соприкасающихся слоев многослойной ограждающей конструкции, ${ }^{\circ} \mathrm{C}$;

- $\delta_{\text {огр }}=\delta_{\text {но }}+\delta_{\text {пс }}+\delta_{\text {мс }}-$ толщина многослойной ограждающей конструкции равна сумме толщин составляющих слоев.

Поступления тепла в подвижной составляющей подвижного слоя компенсируют недостаток суммарного удельного термического сопротивления всех замкнутых слоев $\mathrm{MOK}\left(\Delta t_{\mathrm{R} 3}=\Delta t_{\mathrm{R}}=\Delta t_{\mathrm{R}}\right)$ и затраты на уравнивание температур поверхностей подвижного слоя $\left(\Delta t_{\mathrm{q}}\right)$ с температурами на соприкасающихся с ними поверхностях материала стены и наружного слоя ограждения $\left(t_{\mathrm{McH}}\right.$ и $\left.t_{\text {нов }}\right)$, при учете соответствующих значений и знаков температур на поверхностях подвижного слоя $\left(\mathrm{t}_{\text {псв }}\right.$ и $\mathrm{t}_{\text {псн }}$ ) за счет поступлений в нем от внешних источников при эксплуатации $\left(\mathrm{N}_{\text {псп }}=\mathrm{Q}_{\text {псп }} / \mathrm{t}_{\text {сек }}\right.$, вт).Изображение процесса пересечения МОК, являющегося примером реализации способа регулирования потерь потока через ее поперечное сечение, созданным и перемещающимся в подвижном слое подвижной составляющей, иллюстрируется уравнением перехода температуры этого слоя:

$\Delta \mathrm{t}_{\text {пс }}=\mathrm{t}_{\text {псв }}-\mathrm{t}_{\text {псн }}=\left(\Delta \mathrm{t}_{\mathrm{q}}+\Delta \mathrm{t}_{\mathrm{R}}\right)+\Delta \mathrm{t}_{\text {пс } 3}=\Delta \mathrm{t}_{\text {пс } \Pi}+\Delta \mathrm{t}_{\text {пс } 3}$.

Примечание: на схеме, сплошной вертикальный отрезок $\left(\Delta \mathrm{t}_{\text {псп }}\right)$ подытоживает взаимодействие составляющих подвижного теплового потока, компенсирующих недостаточность удельного термического сопротивления $\mathrm{MOK}\left(\Delta \mathrm{t}_{\mathrm{R}}\right)$ и подаваемого в подвижной среде $\left(\Delta \mathrm{t}_{\mathrm{q}}\right)$, обпспечивающихсответствующую величину составляющей подвижного потока $\left(\Delta \mathrm{t}_{\text {псп }}\right)$ для достижения необходимой температуры на внутренней поверхности подвижного слоя $\left(\mathrm{t}_{\text {псв }}\right): \Delta \mathrm{t}_{\text {псп }}=\Delta \mathrm{t}_{\mathrm{q}}+\Delta \mathrm{t}_{\mathrm{R}}$

Схема, приведенная на рисунке 1 , отображает наиболее экономный вариант регулирования параметров пересечения МОК потоком тепла между ее внутренней и наружной поверхностями, при котором возникновение дополнительных потерь энергии между граничащими слоями ввиду различия температур на поверхностях соприкасающихся слоев, исключено. Это достигается обеспечением равенства температур на поверхностях слоев MOК( см. Рис.: $\mathrm{t}_{\mathrm{мcH}}=\mathrm{t}_{\text {псв }}$ и $\mathrm{t}_{\text {псн }}=\mathrm{t}_{\text {нов }}$ ) за счет поступлений энергии от внешних источников в подвижной составляющей подвижного потока в подвижном слое $\left(\mathrm{q}_{\text {псП }}, \mathrm{BT} / \mathrm{M}^{2}\right)$. При нарушении равенств, представленных схемой, должны возникнуть изменения угла наклона суммарного отрезка, иллюстрирующего изменение температур в подвижном слое, стимулирующие возникновение тепловых потоков между подвижным и замкнутыми слоями МОК, чего следует избежать. 


\section{V. ОСОБЕННОСТИ ПЕРЕХОДА ТЕПЛОВОГО ПОТОКА ЧЕРЕЗ ПОДВИЖНЫЙ СЛОЙ}

Особенностями (дополнительными свойствами, проявляемыми подвижной составляющей подвижного слоя) потока в подвижном слое МОК считаются:

- сохранение способности го замкнутой составляющей оказывать термическое сопротивление переходу тела, пересекающего подвижный слой $\left(R_{\mathrm{x} 3}\right)$;

- перенос теплонесущей среды, транспортирующей дополнительные поступления тепловой энергии в подвижный слой с подвижной составляющей потока;

- возможность перемещения подвижной среды в пределах подвижного слоя и изменения ее теплотехнических характеристик на этапе эксплуатации МОКж

- взаимодействие (энергообмен) поступлений в среду подвижного слоя в составляющий на которые он может быть суперпозиционирован;

- обеспечение возможности компенсации потерь для установления температурного перепада на поверхностях МОК за счет внешних поступлений.

Действующая ДБН [1], ограничивает учетом потерь энергии в слоях из замкнутых потоков и их составляющих, пересекающих МОК, в соответствии с законом Фурье, интерпретированным учебником [4] для многослойных конструкций:

$\Delta \mathrm{q}_{\mathrm{x}}=\Delta \mathrm{t}_{\mathrm{x}} / \mathrm{R}_{\mathrm{x}}$, где: $\mathrm{x}$ - это шифр или номер слоя МОК. Тогда, температурный перепад на поверхностях ограждения $\left(\Delta \mathrm{t}_{\text {огр }}{ }^{\circ} \mathrm{C}\right)$ складывается из потерь температуры на внутренней (в) и наружной (н) поверхностях MOE - $\Delta \mathrm{tB}(\mathrm{H})=\mathrm{q}_{\text {огрх }} \mathrm{R}_{\mathrm{B}(\mathrm{H}),}{ }^{\circ} \mathrm{C}$ и суммы потерь в слоях - $\left.\sum \Delta \mathrm{t}_{\mathrm{x}}=\sum\left(\mathrm{q}_{\text {огрх }} \mathrm{R}_{\mathrm{x}}\right),{ }^{\circ} \mathrm{C}\right): \Delta \mathrm{t}_{\text {огр }}=\mathrm{t}_{\mathrm{B}}$ $\mathrm{t}_{\mathrm{H}}=\Delta \mathrm{t}_{\mathrm{B}}+\Delta \mathrm{t}_{\mathrm{H}}+\left(\Delta \mathrm{t}_{\mathrm{Ho}}+\Delta \mathrm{t}_{\mathrm{мc}}+\Delta \mathrm{t}_{\text {пс }}\right)=\mathrm{q}_{\text {огрх }}\left(\mathrm{R}_{\mathrm{B}}+\sum \mathrm{R}_{\mathrm{x}}+\mathrm{R}_{\mathrm{H}}\right),{ }^{\circ} \mathrm{C}[1-$ 4], где:

- $\Delta \mathrm{t}_{\text {но }}$ - падение температуры в замкнутом наружном слое МОК;

- $\Delta \mathrm{t}_{\mathrm{mc}}$ - падение температуры в замкнутом слое материала стены МОК;

- $\Delta \mathrm{t}_{\text {пс }}$ потери температуры потока в подвижном слое МОК;

Падения температуры в замкнутых слоях $\left(\Delta \mathrm{t}_{\text {но }}\right.$ и $\left.\Delta \mathrm{t}_{\mathrm{мc}}\right)$ и замкнутой составляющей подвижного слоя $\left(\Delta \mathrm{t}_{\text {псз }}\right)$ мотивированы преодолением термического сопротивления каждого слоя должны осуществляются за счет переходящего через МОК потока в соответствии с известными методиками соответствующими требованиям ДБН. Например: $\mathrm{R}_{\text {псз }}=\delta_{\text {пс }} / \lambda_{\text {псз } 3},\left(\mathrm{M}^{2} \mathrm{x}^{\mathrm{o}} \mathrm{C}\right) / \mathrm{\text {вT}}$, тогда $\Delta \mathrm{t}_{\text {псз } 3}=\mathrm{q}_{\text {огрх }} \mathrm{R}_{\text {псз }},{ }^{\circ} \mathrm{C}$.

Но идея устройства специального подвижного слоя с подвижной составляющей, поступлений от внешних источников, позволяет ограничиться при проектировании обеспечением технологичности геометрических и конструктивных параметров замкнутой составляющей $\left(\delta_{\text {пс }}=\delta_{\text {псз } 3}\right.$ и $\left.\lambda_{\text {пс }}=\lambda_{\text {псз } 3}\right)$ для перемещения вдоль оси подвижного потока его подвижной составляющей, пересекающей тепловой поток между поверхностями МОК. При этом, температурные характеристики подвижной со- ставляющей, затребываемые от внешних источников для обеспечения соответствующих параметров поверхностей подвижного слоя при эксплуатации, регламентированы свойствами замкнутой составляющей, созданными на этапе осуществления капитальных затрат: $\Delta \mathrm{t}_{\text {псп }}=\Delta \mathrm{t}_{\text {пс }}-\Delta \mathrm{t}_{\text {псз }}$.

Как следует их представленного материала, расчет значений температур на поверхностях подвижного слоя затруднений не вызывает. Температура на внутренней поверхности подвижного слоя устанавливается и регулируется, как равная температуре потока, поступающего от внешних источников. К сожалению, регулирование температуры на наружной поверхности подвижного потока, требующее стремиться к равенству температур на внутренней поверхности наружного слоя (но), осуществимо только за счет изменения потерь энергии, при заданной температуре на поверхностях подвижного слоя. Теоретические основы решения задачи считаются обе известными. Но для регулирование температур на внутренней и наружной поверхности методических указаний нет и их разработка может стать целью продолжения процесса регулирования потерь потоком, пересекающим поперечное сечение МОК. Составление схемы перехода теплового потока через многослойное ограждение с подвижной составляющей не иллюстрирует на данном этапе изменения мощностей взаимодействующих потоков в период эксплуатации в подвижной среде, ограничиваясь оценкой состояния температурного поля на границах соприкасающихся потоков.

\section{ВЫВОДЫ}

1. Перспективы использования подвижных потоков для регулирования потерь МОК декларированы и обнародованы в действующих нормативных источниках, но приведенные в них указания и приложения к ним, сдерживают внедрение преимуществ, проявляемых подвижными средами при их эксплуатации.

2. Включение подвижных слоев в состав конструктивов МОК позволяет уподобить их теплообменным аппаратом , соответствующим классу международной патентной классификации $\left(\mathrm{MПК}^{12}\right)$ c шифром - F24 9/12. В число функций, выполняемых ими следует добавить решение задачи:"Регулирование потерь тепловыми потоками, пересекающими МОК, поступлениями в подвижных составляющих при их эксплуатации".

3. Важнейшей из теоретический предпосылок, препятствующих использованию свойств и преимуществ подвижных потоков, является основополагающая ДБН концепция постоянства термических сопротивлений МОК.

4. Включение подвижного слоя в конструкцию МОК стимулирует модернизацию способов регулирования потерь за счет перераспределения энергопоступлений на этапе требует пересмотра указанного ДБН описания процесса взаимодей- 
ствия пересекающихся потоков в период эксплуатации.

5.Составление схемы перехода температуры через слои МОК и в ее слоях иллюстрирует перспективность для дальнейшего изучения преимуществ суперпозиционирования подвижного потока МOK на подвижные и замкнутую составляющие, взаимодействующие между собой в потоке подвижного слоя на этапе эксплуатации.

6. Во исключение потерь тепл через внутреннюю и наружную поверхности подвижного слоя и полного использования потенциала внешних слоев температура поступлений должна равняться температурам на поверхностях слоев, граничащих с подвижным.

7. Достижение необходимых температур на поверхностях подвижного слоя регулируется теплообменом в составляющих подвижного слоя.

8. Целесообразно рассмотреть подробно теорию теплообмена между составляющими суперпизиционируемого потока в подвижном слое, дополняю щую оценку изменения температур учетом энергопотерь в переходящем МОК потоке.

\section{ЛИТЕРАТУРА}

1. ДБН В.2.6-31:2006 Тепловая изоляция зданий и сооружений. - К:Минстрой Украины "Укрстройинформ" 2006г., 65 стр., с ил

2. СНиП \|-3-79** Строительная теплотехника м:Госстрой, 1986г., 32ст., с ил.

3. Рекомендации по проектированию навесных фасадных систем с вентилируемым воздушным зазором для строительства и реконструкции зданий. - М:Москомархитектура, 2002г., 104 с., с ил.

4. Тихомиров К.В., Сергиенко Э.С. Теплотехника, отопление и вентиляция. - М: Стройиздат, 1991г., 480стр., с ил.

5. Прусенков Н.А. Капитальные и эксплуатационные затраты ограждений. - Одесса: ВЕСТНИК ОГАСА, выпуск №45, 2012г., ст 199-2002.

\title{
N.A. Prusenkov
}

Odessa State Academy of Civil Engineering and Architecture, Didrihson str., 4, Odessa, 65029.

\section{SCHEME DETERMINING COMPONENTS HEAT LOSS IN A MOVING LAYER OF THE MULTILAYERS WALLING}

\begin{abstract}
Changing attitudes to the regulatory framework of mobility layers walling requires a revision of the calculation scheme for determining losses flow, passing it. This scheme favors the development of ways to avoid them arising

from the temperature difference between adjacent layers on surfaces that are automatically excluded in enclosures consisting only of closed layers. It illustrated the temperature at the boundary alignment layers in the heat input moving component, the moving stream. Description of the method should be supplemented assessment of energy in the limit Lakh moving bed that goes beyond the purpose of publication.
\end{abstract}

Keywords: agile and vicious layers component flow, heat transfer, energy loss

\section{REFERENCES}

1. DBN V.2.6-31:2006 Teplovaya izolyatsiya zdaniy i sooruzheniy. - K:Minstroy Ukrainyi "Ukrstroyinform" 2006g., 65 str., s il

2. SNiP \|-3-79** Stroitelnaya teplotehnika m:Gosstroy, 1986g., 32st., s il.

3.Rekomendatsii po proektirovaniyu navesnyih fasadnyih sistem $\mathrm{s}$ ventiliruemyim vozdushnyim zazorom dlya stroitelstva i rekonstruktsii zdaniy. M:Moskomarhitektura, 2002g., 104 s., s il.

4. Tihomirov K.V., Sergienko E.S. Teplotehnika, otoplenie i ventilyatsiya. - M: Stroyizdat, 1991g., 480str., s il.

5. Prusenkov N.A. Kapitalnyie i ekspluatatsion-nyie zatratyi ograzhdeniy. - Odessa: VESTNIK OGASA, vyipusk \#45, 2012g., st 199-2002.

Отримана в редакції 17.07.2014, прийнята до друку 31.07.2014 